\title{
The Impact of CBSE's Activities on E-Commerce Applications
}

\section{Lena Khaled}

Department of Software Engineering, Zarqa University, Amman, Jordan.

Email: lenaumleen@yahoo.com

Received February $1^{\text {st }}, 2013$; revised March $9^{\text {th }}, 2013$; accepted March $14^{\text {th }}, 2013$

\begin{abstract}
Component based technology is widely used for both academicians and business. There are numbers of benefits for using this type of technology. First, it helps to increase the efficiency and maintainability of software. Second, it improves quality and helps to enhance productivity. Third, the reuse approach that supports component based technology decreases the time to market. Component based e-commerce can be used to solve e-commerce difficulties at application level as well as at system level. This paper introduces how component based activities have effect mainly on building the framework of the e-commerce. It also discusses how the architectural design of the application can be synthesized.
\end{abstract}

Keywords: Component; Component Based Activity; CBSE; Agent Software Model

\section{Introduction}

Electronic commerce (e-commerce) defines the process of selling, buying and exchanging products, services and even information through networks. E-commerce can be defined from different points of view. For example, in the business view; it is defined as doing business electronically by completing all the services over electronic networks. Therefore, a broader definition for commerce, which is called electronic business, is used. In addition to buying and selling goods and services that are made by e-commerce, e-business includes servicing customers, collaborating with business partners [1]. E-commerce is always changing the area of business management and information technology; it is the scope that is always upto-date. Because of this change and its impact on society, organization and customers, it is very important to study how to increase the product's efficiency and produce high productivity with low cost and less time to market.

In order to make e-commerce successful, we need to use a good paradigm for such type of applications. This paper describes managing such applications through component based paradigm. This type of paradigm provides companies with high speed applications to compete with others through the internet. This paper is organized as follows: Section 2 describes related works, and it also describes all authors who have worked on such area and then their works are compared to this paper. Section 3 defines component, Component Based Software Engineering (CBSE), as well as it shows the activities of CBSE. Section 4 describes the case study that is used in this paper. Section 5 shows the result. The discussion and conclusion are done through Sections 6 and 7.

\section{Related Works}

Many researchers worked on building a model of component based e-commerce systems. Reference [2] proposed a new method for building a formal model for component based e-commerce system using hierarchal structures to organize design schemes in many levels of the e-commerce system. They also analyzed the modeling process of the payment system as a case study of their work. Reference [3] introduced the requirements that an architecture should meet for it to be appropriate for marketplace. They examined UNIX filter and Visual Basic components then presented two graphical interfaces for components; one used by the end user and the other one used by the designer. The third paper, [4], worked on created components for mobile commerce with the emphasis on many storage and retrieval components. Many tasks were carried out. First, existing models were used to define a model for component based mcommerce (mobile commerce), second a UDDI (Universal Description Discovery and Integration) was used to model component repository. They concluded that the proposed repository could not be compared with the existing repository architecture because it was not implemented, UUDI was used instead of that. Reference [5] presented a data modeling approach that linked the process and the document at the same time. It took into account the different stakeholders requirements and the 
result of the paper was a comprehensive library of reusable business information entities that has been developed to easily model new business documents to ensure an efficient growth of the business document library. As from previous works the component model was adopted to show how requirements are gathered and refined to build architectural model for the system, but this work describes the component based activities in details and then shows how these activities affect mainly building systems such as e-commerce systems. It also describes the effectiveness of the quality of the produced system as well as the delivered time of it through using such activities.

\section{CBSE Technology}

CBSE is an approach of software development which is based on reusing software components. For custom services, CBSE is an effective way to develop a new enterprise system. It has become the most important software development because software systems are becoming more complex and the customer needs a software that is delivered and deployed in short time. The only way to deal with this complexity is to reuse rather than to re implement software components[6].

\subsection{Component Definition}

A software component is an independent piece of executable software and is executed as a unit that is only accessed through their interfaces. Therefore, it must be possible to obtain details of their interfaces [7].

Any component has three main features [8]:

- It is an independent part of a system that submits a specific function;

- It works in the framework of a specific architecture;

- Each component communicates with other component by its interface.

To ensure that component base run in an efficient manner, we must focus on second feature, the framework of the architecture, which is represented as layers.

\subsection{Activities of CBSE}

CBSE is based on the reuse approach which must find and integrate reusable components. The main idea of the component-based activities is building systems from already existing components. Different models described the life cycle of CBSE, like Somerville's model, models proposed by Kotany et al. and Y model. The general process aims to gather and evolve building blocks which are called components. This paper adopts Somerville's model which follows the sequential approach [9]. Figure 1 shows the principle activities that are:

- Outline requirements: In the beginning, the user requirements are developed in an outline rather than in detail. If the requirements are too specific, then the components that are going to be used to meet these requirements are very limited. As a result, the complete requirements need to be defined in order to define the components of the system.

- Refining and modifying the requirements: This is done early in the process, depending on the available components. During this stage the requirements of the component are analyzed and modified to reflect the available components. When it is impossible to modify components, the activity of component analysis may be re entered to search for alternative solutions.

- Architectural design: The architectural design of the system is built. A further search and refining design activity is done through this stage.

- Development: Is the integration of the discovered components. This includes tasks that adjusting components to their specific environment.

The above stages are the basic activities that distinguish CBSE from other software developments [6].

The main idea of the component-based activities is to reuse the available components rather than implementing them. For this reason the availability of existing components must be considered in the requirements and design phases.

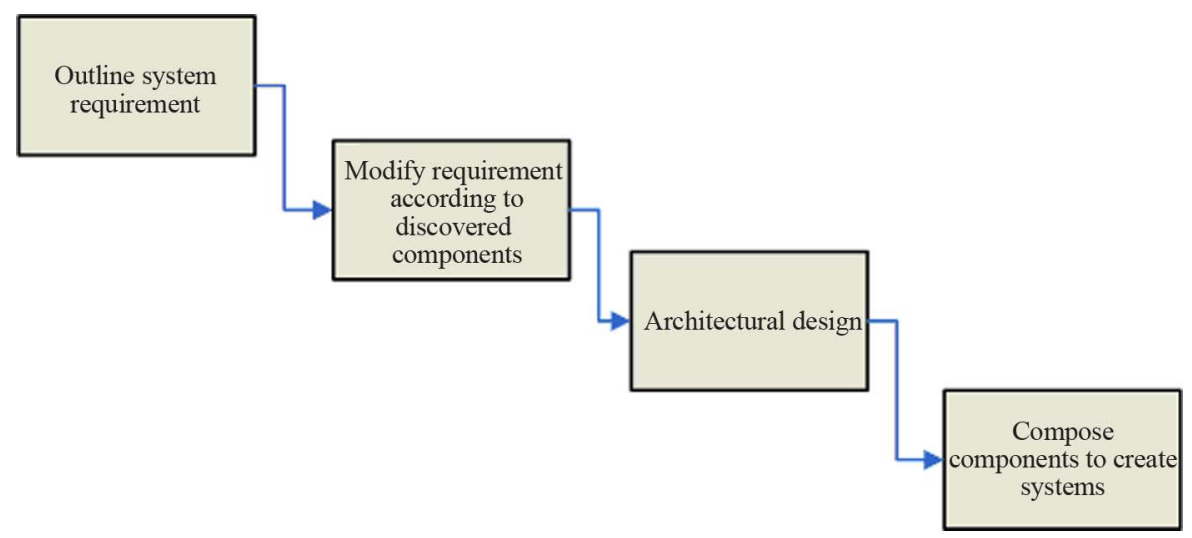

Figure 1. The basic activities of CBSE. 


\section{Case Study}

This paper adopts Job Agency System (JAS) as a case study because of a large number of components introduced, it is one type of Business-To-Consumer commerce (B2C). This type of system is particularly serving technology oriented companies and workers because they use internet repeatedly. Many of job agencies are effective on the web, they used their own web pages to post their jobs, on the other side the job seeker put his resume. In spite of many advantages for job seeker such as finding jobs on a large number of jobs world wide, the main limitations are:

- The fact that many people do not use internet;

- Security and privacy.

From Figure 2 this type of system serves those who are looking for jobs, so the main scope of this system is connecting those people with the recruiters who are looking for employees with specific skills. It is also responsible for transactions of services from business individuals. The employee in electronic JAS has the ability to compare between different jobs, unlike an employee in traditional JAS.

\section{The Result}

One of the most common reasons for using component based technology in e-commerce applications is the concept of distribution i.e. components can be easily distributed through applications, like in shipping goods from one to another. Component based technology provides the best solution for building the structure of applications. The following section describes how component based activities are used to build e-commerce applications and how this type of paradigm affects delivering such type of products.

\subsection{Building E-Commerce Applications from CBSE Activities}

Extracting the product of e-commerce application from the methodology of the component based activities is shown in Figure 3.

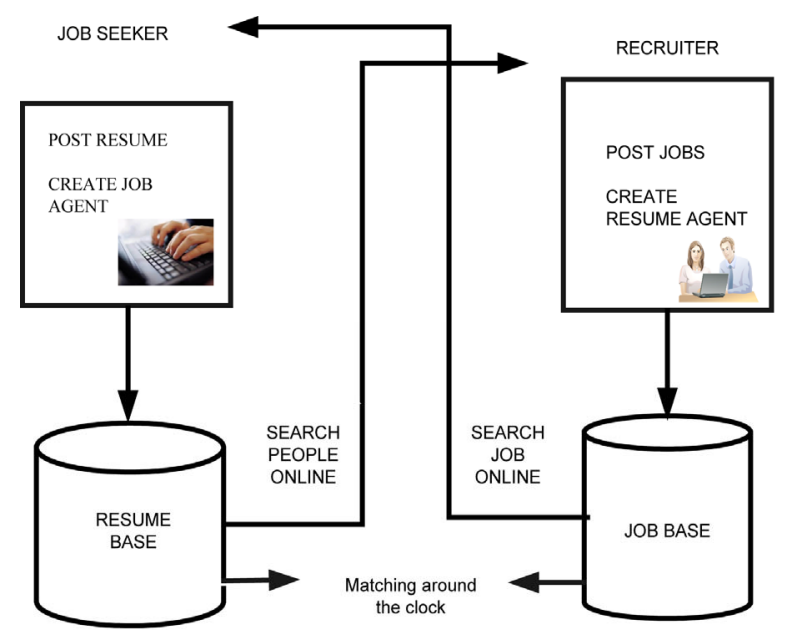

Figure 2. The work of JAS.

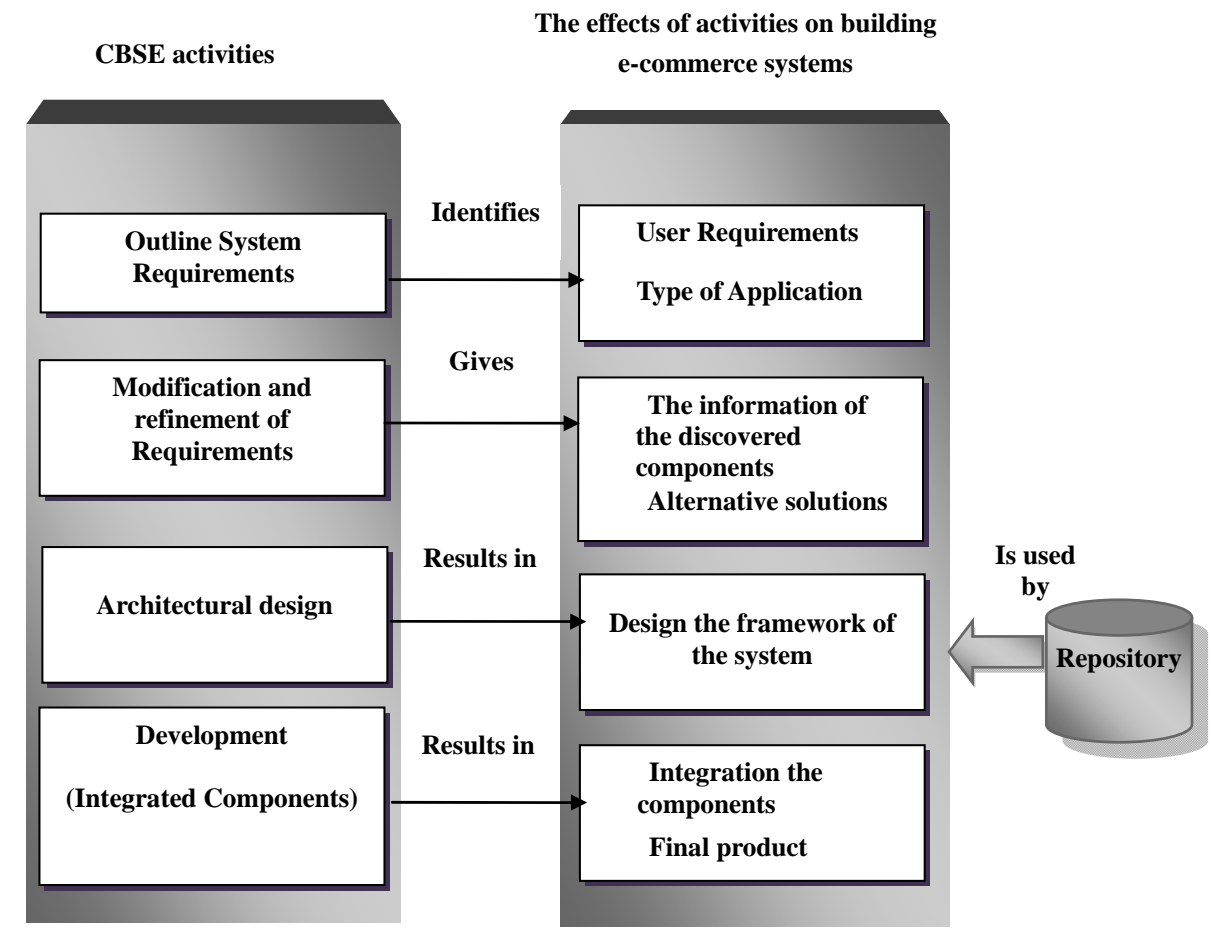

Figure 3. Building e-commerce application from CBSE activities. 
- Specifying requirements is the phase that comes before the first stage of component based activities. In this phase we must specify the user requirements of the system. In requirement specification, we need a complete set of requirements in order to identify the components to reuse. Thus, the user and system requirements should be easy to understand, clear, unambiguous, complete and consistent.

- Outline system requirements is the first stage of component based activities. In this stage, a search for components is made in order to implement requirement specifications in the previous stage. Moreover, the type of system must be specified such as JAS is a one type of B2C commerce and non-functional requirements (set of qualities) must be specified too. The most important qualities that we want to achieve here are reliability, availability, and usability, which is the most essential quality since the user interface must be understandable and easy to navigate. As in Figure 3 this stage identifies user requirements as well as the type of application user.

- Requirement modification is the second stage of the component based activities, in which the requirements are modified depending on the available components. If the user cannot be satisfied from the available components, then re-entered components must be used to search for alternative solutions and this is one of the main strong points in this activity. As in Figure 3 this stage gives the information of the dis- covered components as well as the alternative solutions.

- Architectural design is the third stage of the component based activities. The first step of designing the system is done through this stage. The architectural design synthesis of JAS case study is represented in Figure 4 [10]. As in Figure 3 this stage results in designing the framework of the system.

Figure 4 shows that jobseeker and recruiter are connecting and because of this connection the role of agent appears. Following section defines agent and its role.

- Development (or integrated components) this depends mainly on suppliers which provide components according to their properties. Here the certification documents these properties. Reference [11] provides a prototype tool for the integration phase which consists four parts: broker, supplier and integration and certification body. This is shown in Figure 5. As in Figure 3 this stage results the integration of components then the final product is produced.

Broker in Figure 5 supports both system integrator and supplier by proposing a component database. The broker helps integrator defines its functional requirements and according to this definition, the broker establishes a set of components to the integrator and all this is done through a certification body that associated with each component. The standardizations that embodied in component model facilitate the integration of the component.

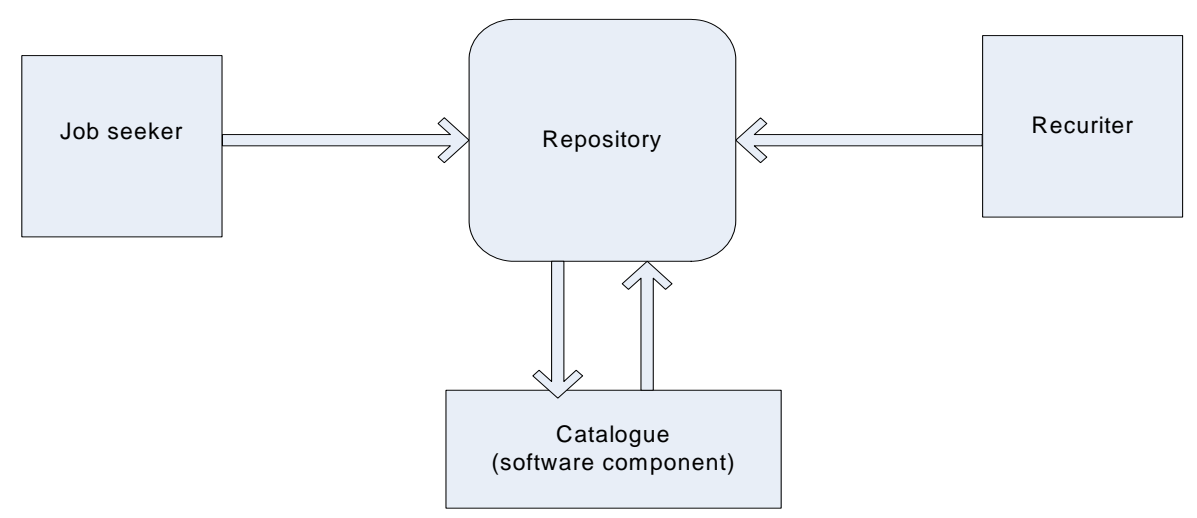

Figure 4. Architectural synthesis of job agency system(JAS).

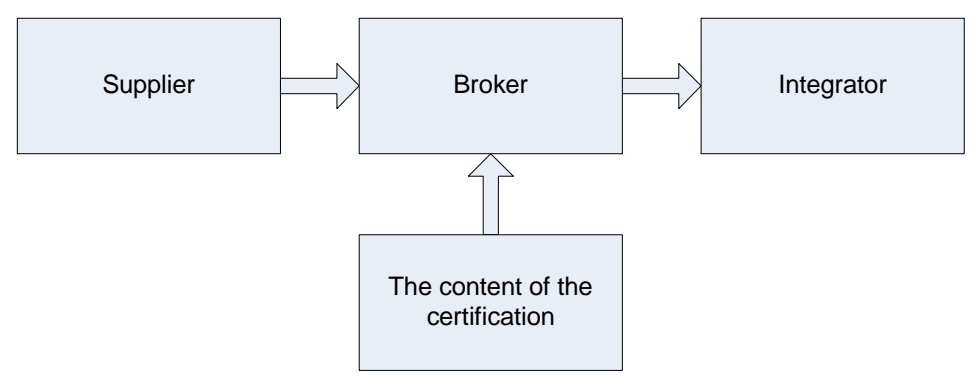

Figure 5. A prototype of integration phase. 


\subsection{Components and Layers}

Because of the large number of jobs and resumes, the importance of intelligent agents appear. An Intelligent agent can be defined in this work as a software component which is capable to finish tasks on behalf of its user [12]. It can help the user to do different tasks such as search and negotiation. Using this type of components decreases the time to search, thus improving the effect of quality on the work. Another important concept used in this architecture is the term catalogue which is defined as software component used for business process. It consists of a set of web pages that describe specific jobs and their organizations and, sometimes, have embedded pictures along with them [8]. Catalogue management must provide ways for defining, retrieving and storing e-catalogues, while supporting multiple views and vocabulary to clearly support the different processes of e-commerce. This type of components provides a flexibility attribute for ecommerce applications. As it is shown in Figure 2, repository communicates with this stage. Repository is used to store the components for suitable access according to the user's requirements [13]. In general, business components are used in e-commerce applications to provide the services of transactions while system components are used for the main services of the application such as storing data and communications. The framework architecture of component, which an e-commerce system must worked on, consists of three tiers and illustrates in Figure 6.

Presentation tier: It must contain the component that is responsible for the usability of the system. Job market catalogue, that contains a set of web servers, is stored here.

Application tier: This contains all the processing that is needed by the electronic market such as e-commerce transaction settlement. This tier is where the application's

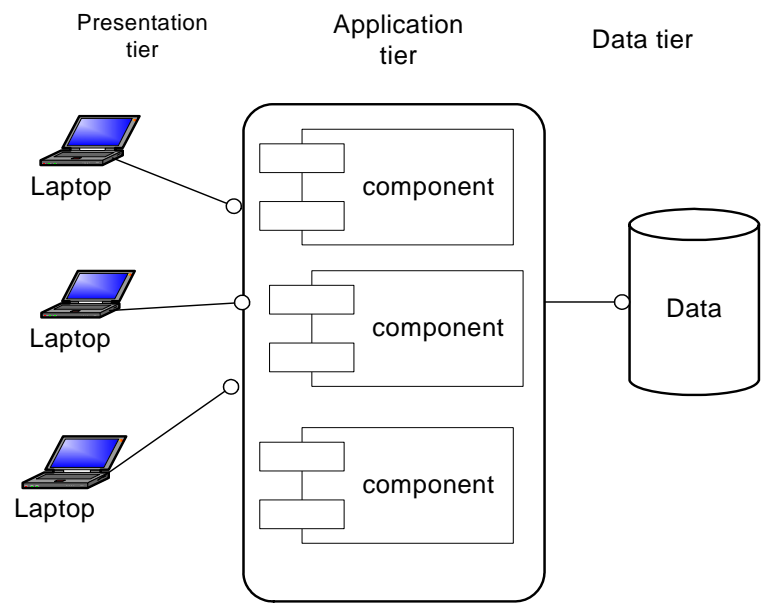

Figure 6. Architectural framework of component based technology. logic locates, so it is often known as business logic.

Data tier: Here is where the data are usually stored and retrieved. This tier can be either a relational database or an object oriented database.

\section{Discussion}

The final product that produces from the final stage of life cycle must be with high level of quality because of the characteristics of integrated components which are:

- Standardization: The standardization of the component means that every component used in the activeties of component based must comply with a standard component model; this model may define the interface of the component, the composition with other components and the deployment of the component in the system. So because of this standardization the final product is with high quality product;

- Certification of the component: The certification supports users that made users decide weather or not the components are according with their requirements, the scheme of the certification provides product trust worthiness and flexibility attribute;

- Reuse approach: As a component technology supports reuse approach this decreases the time to market of the product.

\section{Conclusion}

E-commerce is the infrastructure of doing business. Because the categorizations are different from sell side to buy side in this type of application, managing the complexity is the most important thing to deal with. Dealing with complexity needs a paradigm to build that kind of applications. This paper shows how component based activities affect building such type of applications. Such paradigm provides companies with the speed and the agility that they need in order to be able to compete on the internet.

\section{REFERENCES}

[1] E. Turban, et al., "Electronic Commerce: A Managerial Perspective,” Person Education, Inc., Upper Saddle River, 2004.

[2] X. L. Jin and H.-D. Ma, “An Approach to Formally Modeling the Component Based E-commerce System,” Preceeding of the IEEE International Workshop on Service Oriented Software Engineering, Beijing, 20-21 October 2005, pp. 15-22. doi:10.1109/SOSE.2005.4

[3] E. Whitehead, J. Robbins, N. Medividovic and R. Taylor, "Software Architecture: Foundation of a Software Component Marketplace,” Proceedings of First International Workshop Architectures for Software System, Held in Cooperation with ICSE-17, Seattle, April 1995, pp. 276-282.

[4] Kunene and H. Pinky, "Enterprise Component Archi- 
tecture for Mobile Commerce Services,” University of Zululand Institutional Repository, 2004. http://hdl.handle.net/10530/1046

[5] T. Janner, et al., “A Core Component Based Modeling Approach for Achieving E-Business Semantic Interoperability," Journal of Theoretical \& Applied E-Commerce Research, Vol. 31, No. 3, 2008, pp. 1-16.

[6] I. Somerville, "Software Engineering," Pearson Education, USA, 2011

[7] K. Whitehead, "Component Based Development, Principles and Planning for Business Systems," Pearson Education, UK, 2002.

[8] X. Cai, M. R.-T. Lyu, K.-F. Wong and R. Ko, "Component-Based Software Engineering: Technologies, Development Frameworks, and Quality Assurance Schemes," Software Engineering Conference, 5-8 December 2000, pp. 372-379. doi:10.1109/APSEC.2000.896722

[9] G. A. Ansari, S. A. Mohamed and M. P. Syed, "Modeling of a Component-Based Software through UML Using Object-Oriented Approach,” The IUP Journal of Systems Management, Vol. 9, No. 3, 2011, pp. 42-53. http://ssrn.com/abstract

[10] L. Khaled, “Architectural Design Activities for JAS,” International Journal of Computer Science and Information Technology, Vol. 6, No. 2, 2009, pp. 1947-5500.

[11] J. Boegh, "Certifying Software Component Attributes," 2006. www.computer.org/software

[12] W. Liang, "The Assessment of Intelligent Agent in the B2C E-Commerce Negotiation," Proceedings of the International MultiConference of Engineers and Computer Scientists, Hong Kong, 16-18 March 2011.

[13] J. Sharp and Sh. Ryan, "A Theoretical Framework of Component-Based Software Development Phases," The Database for Advances in Information Systems, Vol. 41, No. 1, 2010, pp. 56-75. 